\title{
Stages of technological process of high anisotropy formation of magnetic properties under «dry» pressing of powder products
}

\author{
Yuri Vernigorov, Valeriy Lebedev*, Irina Davidova, Lydianna Chunakhova and Yuri \\ Korolkov \\ Don State Technical University, Rostov-on-Don, Russia
}

\begin{abstract}
The article proposes the description of technological production process of anisotropic products from powders of high materials which provides powders preparation and products made of them. It has been shown that the main drawback of «wet» pressing technology is low accuracy at dosing wet charge. To eliminate warping and cracking of briquettes obtained by «wet» pressing during the sintering, they are subjected to air drying at room temperature within 48-72 hours. However, the percentage of product failure due to mechanical and magnetic characteristics remains high. Practically all the drawbacks of «wet» pressing can be eliminated by reverse to dry powder pressing, which allows to obtain low mass magnets without additional final finishing machining, to avoid warping and cracking of the product, and to reduce sintering time. Nevertheless, under the dry powder pressing the magnetic characteristics of products are significantly lower than under the wet pressing. When working with disperse materials, a promising direction is to converse them into fluidized state. To obtain the fluidized state of powder materials, vibrational technologies are used, involving the transfer of a wide range of energy to the powder material, i.e. mechanical, acoustic, electric and magnetic energy.
\end{abstract}

\section{Introduction}

At manufacturing products from ferromagnetic materials possessing anisotropy of magnetic properties, powders with particle size close to one-domain are used, which have external magnetic flux, which leads to strong interparticle interaction of magnetostatic nature. The process of micro aggregates (clusters) formation is enhanced due to cohesive interaction of particles. In natural clusters, a macro-vortex ordering of magnetic moments of separate particles [1] is formed and the magnetic moment of the cluster is turned out to be far less than the sum of magnetic moments of its particles. Texturing of powder products in a constant magnetic field increases the clusters stability and does not allow to obtain a high degree of magnetic moments orientation during «dry» pressing. Thus, in «dry» pressing, the magnetic moments of clusters, rather than separate particles, are oriented in the external magnetic field. Besides, it is practically impossible to ensure equal distribution of dry

\footnotetext{
${ }^{*}$ Corresponding author: va.lebidev@yandex.ru
} 
powder of magneto-rigid material by volume in the cavity of the complex mold. Obviously, the increase in the magnetic characteristics of powder products can occur as a result of destroying the powder clusters before texturing.

When working with powder materials characterized by high forces of interparticle interaction, the most promising direction of attenuation of interparticle interaction and destruction of powder clusters is the creation of a magneto-vibrational layer. The dispersed system of ferromagnetic material placed in the interpolar space of alternating current electromagnet under certain conditions can be put into the mode of stable parametric variations. Such an ensemble of particles oscillating under the influence of electromagnetic field should be considered as a space of the magneto-vibrational layer (MVL). Technological capabilities of magneto-vibrational technology based on application of alternating magnetic field make it possible to reduce the milling time, which requires large expenses of total energy; to implement powder dosing in automatic mode and to make transition to automated production [2].

\section{Problem Statement}

Determination of technological processing sequence during the pressing of powder anisotropic products with the application of magneto-vibrational layer.

\section{Purpose of the Study}

To obtain high magnetic texture in a powder product, it is necessary to implement two main processes: to destroy to separate particles natural micro aggregates having macro-vortex ordering of the magnetic moments of the particles, if possible and to create before pressing a parallel system of magnetic moments of separate particles.

1. Let mass $M$ be defined by the condition of the magnetic cluster destruction, $I-$ by the moment of inertia, and $\mathrm{P}_{\mathrm{m}}$ by the magnetic moment in inhomogeneous magnetic field $\vec{B}=$ $\overrightarrow{B_{c}}+\overrightarrow{B_{V}} \cos \omega t$. Moreover, the amplitude of the variable field $B_{V}$ is small, but the field is very heterogeneous. Suppose the variable field induction gradient along the $O Y$ axis is advantageous. Power lines of constant and variable magnetic fields are mutually perpendicular. The motion of the magnetic cluster is described by the equations:

$$
\begin{aligned}
& M \overrightarrow{r^{\prime \prime}}=\left[\overrightarrow{P_{m}} \frac{d \overrightarrow{B_{V}}}{d y}\right]-k \overrightarrow{r^{\prime}} \\
& I \overrightarrow{\varphi^{\prime \prime}}=\left[\overrightarrow{P_{m}} \overrightarrow{B_{V_{0}}}\right]+\left[\overrightarrow{P_{m}} \overrightarrow{B_{c}}\right]-\mu \overrightarrow{\varphi^{\prime}},
\end{aligned}
$$

where $\overrightarrow{B_{V_{0}}}=\overrightarrow{B_{V}} \cos \omega t, \mathrm{k}$ and $\mu$ - coefficients of medium resistance to translation and rotational motion.

Assuming that at $\mathrm{t}=0$ the cluster rests, the initial conditions are as following: $r(0)=$ $r^{\prime}(0)=0, V(0)=V^{\prime}(0)=0$. Equations (1) were written assuming that the magnetic moment of the cluster $\overrightarrow{P_{m}}=\sum_{i=1}^{n} \overrightarrow{P_{l}}$ is composed of the magnetic moments of its constituent particles. Considering the conditions of particle separation from the cluster $[3,4,5]$, we obtain values of magnetic field induction gradient required for cluster destruction:

$$
\frac{d \overrightarrow{B_{V}}}{d y} \geq \frac{\mu_{0} f_{i} \overrightarrow{P_{m}}}{4 \pi r R(r+R)^{2} \cos \varphi}
$$


where $f_{i}$ - the coefficient of statical friction between parts of the cluster; $r$ and $R-$ are equivalent radii of a small particle and a cluster, $\varphi$ - is the angle between the vertical axis and the magnetic moment $\overrightarrow{P_{m}}$.

The advantage of inhomogeneous magnetic field at the destruction of clusters of dispersed magneto-rigid ferromagnets is determined by the fact that within inhomogeneous magnetic field the field energy is pumped directly to translational degrees of freedom. Inside homogeneous field, the field energy is pumped to the rotational degrees of freedom of the particles and only then is transferred to the translational degrees of freedom due to mechanical interaction. Firing-pin mechanism of destruction of aggregates, embedded in the basic model, leads to the advantage of inhomogeneous field.

2. Under certain values of magnetic field induction, a magnetic structure is formed in the magneto-vibrational layer: at first in the form of local linear formations consisting of several tens and hundreds of particles, and then in the form of equidistant circuits located along the field power lines. It can be assumed that the transition of the dispersed system from the magneto-vibrational layer to the «solid» phase will occur provided that the particles stop back and forth motion. Disperse medium can form ordered highly cooperative structures due to the fact that external constraints keep the system away from equilibrium $[6,7]$. At the same time, a new principle of ordering is manifested, different from the Boltzman principle of chaotization. We find the transitional condition by equating the energy of translation and rotational movement of particles with the energy of interaction of particles with a permanent field $[8,9]$ :

$$
B_{C}=\frac{P}{2 \pi \omega}\left(\frac{B_{v}^{2}}{I}+\frac{1}{m}\left(\frac{\partial B_{v}}{\partial y}\right)^{2}\right)
$$

Expression (3) makes it possible to calculate the induction of a permanent magnetic field at which the dispersed system changes into a pseudo-solid phase (Fig. 1). The circuits formed from the particles make transverse oscillations in the inhomogeneous magnetic field. The forces of magnetostatic and adhesion interaction, holding the particles in the circuit, play the role of quasi-elastic forces, which allows these circuits to be considered as elastic strings. The impact force acting from the side of inhomogeneous field changes simultaneously along the entire length of the magnetic string and excites transverse oscillations of only the basic tone

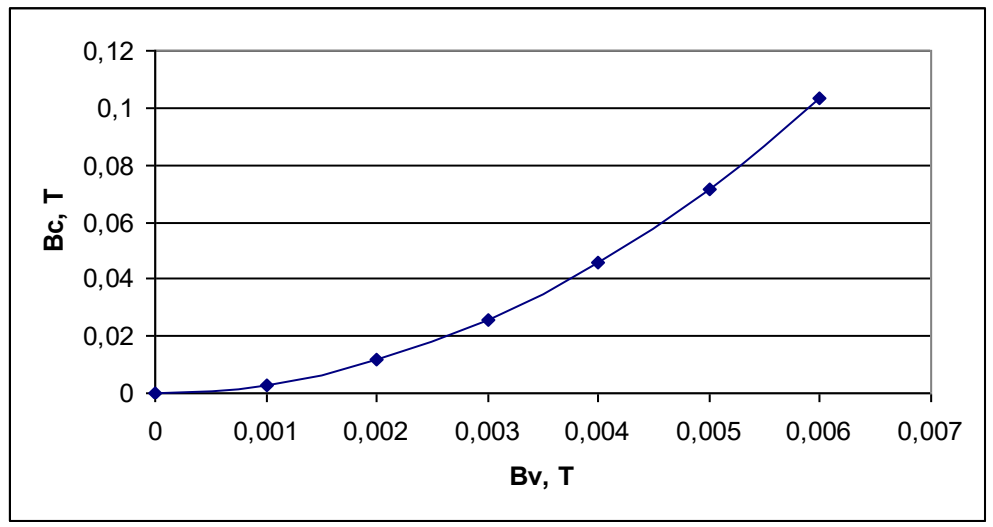

Fig. 1. Ratio of magnetic field parameters at the transition of dispersed system from magnetovibrational layer to pseudo-solid phase at $r=100 \mu \mathrm{m}, \frac{d B}{d y}=2 \mathrm{~T} / \mathrm{m}, \mathrm{P}=5 \cdot 10^{-10} \mathrm{~A} \cdot \mathrm{m}^{2}, v=50 \mathrm{hz}$.

Suppose that in the equilibrium position the magnetic string coincides with the axis $\mathrm{OX}$ and the direction of the power lines of a permanent field, then we get the following equation of small transverse vibrations: 


$$
m \ddot{y}+\beta \dot{y}-F\left(B_{c}\right) \frac{\partial^{2} \phi}{\partial x^{2}}=P \frac{\partial B_{v}}{\partial y} \sin \phi \cos \omega t,
$$

where $m$ and $P$ - are the mass and the magnetic element of the string $d l, \beta$ - is the coefficient of resistance to the string motion, $F\left(B_{c}\right)$ - is the force of string tension, $\varphi-$ is the angle between $\vec{P}$ and OY axis. $\vec{B}_{c}$ и $\vec{B}_{v}$ - are the inductions of permanent and variable constituents of magnetic field.

Considering boundary and initial conditions $y(0, t)=y(l, t)=0 ;\left.\quad y(t)\right|_{t=0}=$ $\left.y^{\prime}(t)\right|_{t=0}=0$ solution for the basic mode of oscillations has the following form

$$
y(t)=\frac{2 D_{0}}{l \delta \omega} \frac{\sin (\omega t-\alpha)}{\sqrt{1+Q_{1}^{2} \gamma_{1}^{2}}},
$$

where $\quad D_{0}=\frac{P}{m} \frac{\partial B_{v}}{\partial y} \sin \phi \quad \delta=\frac{\beta}{m} ; Q_{1}=\frac{\omega_{1}}{2 \delta} ; \gamma_{1}=\frac{\omega}{\omega_{1}}-\frac{\omega_{1}}{\omega} \quad \omega_{1}-$ is the frequency of natural oscillations of the magnetic string, $l-$ is the length of the string, $\alpha=\operatorname{arctg}\left(Q_{1} \gamma_{1}\right)$.

3. On the basis of studies of the mess-order transition in the magneto-vibrational layer and established patterns of obtaining spatial stability of powder particles corresponding to ferromagnetic ordering, a method of texturing of highly anisotropic products under the pressing of dry powder has been developed. Magneto-vibrational technology of formation of magnetic structure of powder magnets involves the implementation of the following stages:

1. At the first stage (Fig. 2), by selecting values of magnetic induction amplitude, magnetic field frequency and field action time, powder clusters are destroyed due to their mutual impact. Between 4 and 7 seconds, the maximum intensity of powder movement in the mold was achieved.

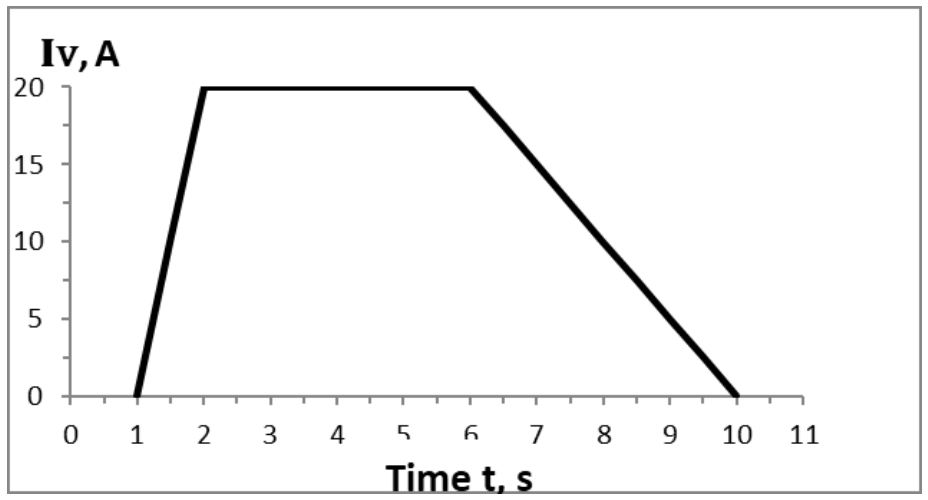

Fig. 2. The change of the force of alternating current with the flow of time.

2. At the second stage (Fig. 3) induction of permanent field constituent is increased, and the magnetic moments of particles are oriented along power lines of magnetic field. Further increase of $\mathrm{Bc}$ leads to the formation of the structure of magnetic circuits. The maximum value of direct current between 9 and 11 seconds of the process in the gap between the punches led to the fixation of magnetic strings. Since most clusters have previously been destroyed, circuits are formed predominantly from the separate particles. By increasing the induction of the permanent magnetic field, we form a structure from magnetic strings, by providing a mode of their resonance oscillation. 


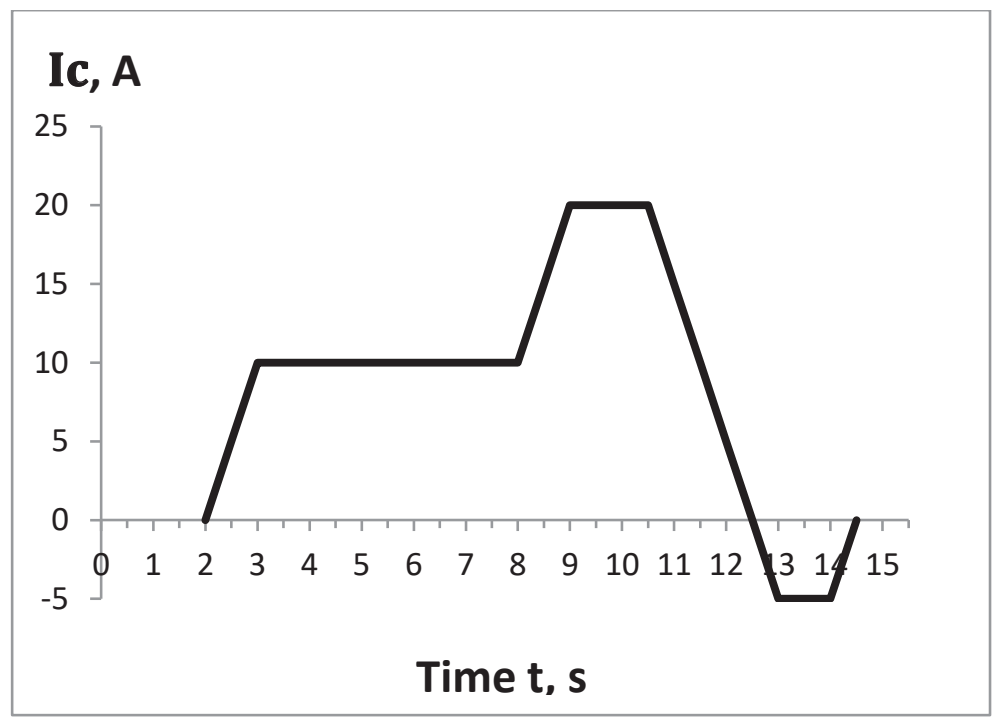

Fig. 3. The change of the force of direct current with the flow of time.

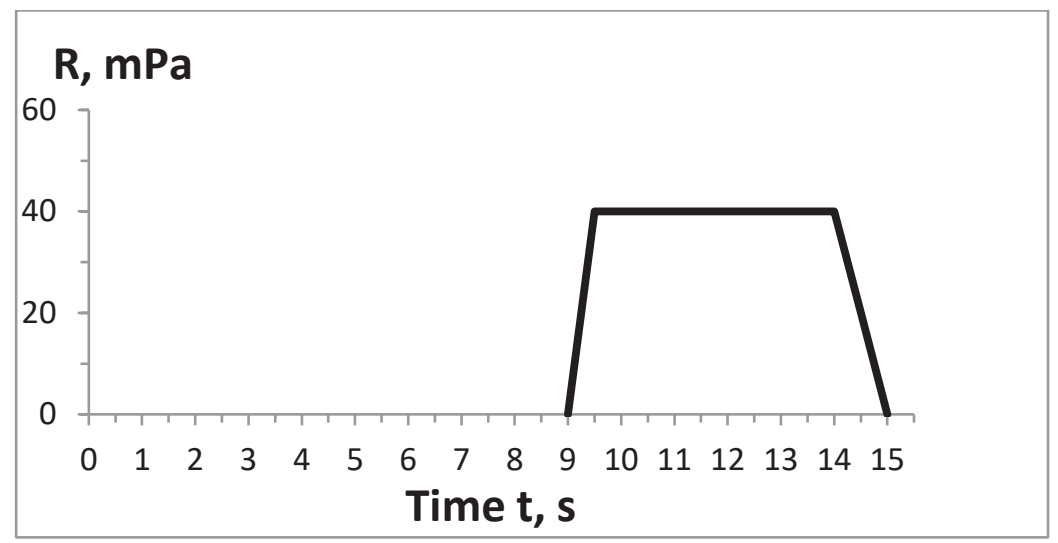

Fig. 4. Cyclogram of mechanical action during pressing.

Further increase of constant component of magnetic field induction leads to fixation of magnetic circuits. Then, after turning off the variable field, pressing is performed (Fig. 4). Afterwards, the sample was demagnetized (a demagnetizing field was created in the matrix between 13 and 15 seconds), pressed out and sintered.

The process of forming a system of magnetic strings from a dispersed medium depends on the field frequency. In particular, the change of the field frequency while maintaining the remaining electromagnetic field parameters permanent leads to an increase of residual magnetic induction, coercive force and reaches its maximum at the frequency of $300 \mathrm{~Hz}$ for cylindrical magnets with a diameter of $9.1 \mathrm{~mm}$, and a height of $2 \mathrm{~mm}$.

4. The principle of operation of the installation, which ensures the formation of magnetic anisotropy of powder products in an alternating magnetic field, is obvious from Fig. 5. Magnet coils (4) installed on the plate and slide of hydraulic press created permanent magnetic field. The alternating current electromagnet had a stacked core; the shape of the pole pieces (3) provided an alternating field of a given topology and an induction gradient of up to $1.6 \mathrm{~T} / \mathrm{m}$. 


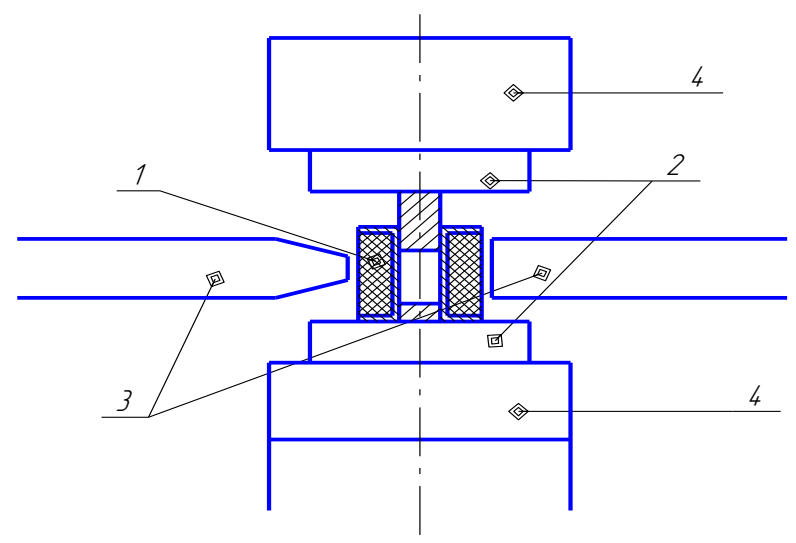

Fig. 5. The scheme of pressing knot with application of magneto-vibrational layer. 1 - mold; 2 backer; 3 - pole-pieces of alternating current electromagnet; 4 - coils of permanent current electromagnet

The mold (1) was placed on the hydraulic press plate. The force lines of the permanent magnetic field are directed along the vertical axis of the mold, the alternating field was oriented perpendicular to the lines of the constant field. Molds of various designs were used depending on the range of magnets to be pressed. The general requirements for all molds used in magneto-vibrational technology are as follows:

- working part of mold must be made of material, strength of which provides its long service life and helps to reduce the losses of electromagnetic power on eddy currents;

- working volume must exceed the free bulk volume by 4-5 times in order to effectively use particle impact processes when the powder enters the state of magneto-vibrational layer.

The process proceeded as follows. The lower punches were immersed in the mold and the powder was spread. Next, the matrix volume was locked with the upper punch and magnetic fields were turned on according to the cyclogram (Fig. 1-4). The "shelf», depending on $I_{c}(t)$ between 4 and 8 seconds, allows to obtain the maximum intensity of powder movement in the mold matrix by creating an induced magnetic moment in the cluster. The dependence $I_{v}(t)$ ensured a fairly fast creation of high-intensity magnetovibrational layer from powder, the process of forming an equidistant system of magnetic strings and their resonant oscillations between 8 and 9 seconds.

Between 4 and 7 seconds the maximum intensity of powder movement in the mold matrix was achieved. The maximum rating of direct current between 9 and 11 seconds of the process in the gap between the punches led to the fixation of magnetic strings. Between 13 and 15 seconds, a demagnetizing field was created in the matrix.

By using a thin-walled mold (Fig. 6), a magneto-vibrational layer was created, an anisotropic structure was formed, and a pressing was performed in one matrix. 


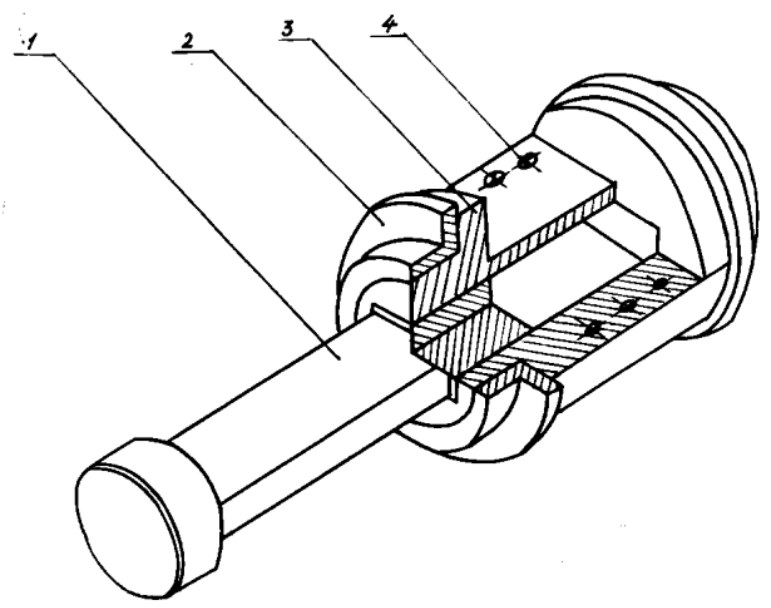

Fig. 6. A thin-walled mold. 1 - punch; 2 - ring; 3 - matrix; 4 - bolt.

\section{Findings}

The impact assessment of electromagnetic field parameters on the properties of the examined samples was carried out in several stages. At the first stage the induction of a permanent magnetic field where clusters are formed was determined. The induction of the field has been found to be substantially dependent on the powder material and the size of its particles. At the second stage, a magneto-vibrational layer was formed in the mold from the dry charge; afterwards it was textured with a permanent magnetic field and, finally, pressed. Magnetic parameters of green blanks were measured. It was determined that with an increase in the induction gradient of an alternating magnetic field to $1.5 \mathrm{~T} / \mathrm{m}$, the magnetic characteristics increase; with a further increase in its residual induction and coercive force change slightly, but the quadracy of the loop continues to increase. The preliminary creation of the magneto-vibrational layer from the dry powder resulted in an increase in residual induction by $10 \%$, a magnetization coercive force by $5 \%$ and a loop rectangle coefficient by $6 \%$, which can be explained by the destruction of clusters in the magneto-vibrational layer.

Pressing was carried out according to the method «up to the stop against the limiting ring» herewith the density of shaped ferritbarium blanks of $3,2-3,5 \mathrm{~g} / \mathrm{sm}^{3}$ was provided. Forging pressure of $40 \mathrm{mPa}$ was applied in the direction of the permanent magnetic field lines. Sintering was carried out in air at $1200^{\circ} \mathrm{C}$ within two hours. Shrinkage of magnets across the texture is $12-13 \%$, parallel to the texture - up to $20 \%$. Density of the baked samples measured by the hydrostatic way in octane changed within $4,53 \div 4,69 \mathrm{~g} / \mathrm{sm}^{3}$.

It was found that the process of forming a system of magnetic «strings» from dispersed medium depends on the frequency of the field. In particular, changing the field frequency at maintaining the remaining electromagnetic field parameters constant leads to an increase in residual induction $\mathrm{B}_{\mathrm{r}}$, coercive force $\mathrm{H}_{\mathrm{cj}}$ and reaches its maximum at frequency of $300 \mathrm{~Hz}$ for cylindrical magnets with a diameter of $9.1 \mathrm{~mm}$, and a height of $2 \mathrm{~mm}$. Micro engine stators made according to the magneto-vibrational technology from barium ferrite powders with a specific surface area of 0,67 to $1,20 \mathrm{~m}^{2} / \mathrm{g}$ had a density of about $4,4 \mathrm{~g} / \mathrm{sm}^{3}$ and a residual induction on the working surface from 0,053 to $0,042 \mathrm{~T}$. 


\section{Conclusion}

The use of dry powder is an important feature of the method. The method allows dosing the initial material and obtaining magnets of low mass without additional finishing treatment, avoiding warping, cracking, etc. Magneto-vibrational technology allows to reduce the sintering time from 74 hours at «wet» pressing to 5-6 hours. Large technological opportunities of magneto-vibrational technology are offered due to relaxation in the requirements to the quality of charge material, in particular to its granulometric composition. This makes it possible to reduce the milling time, which requires large expenses of total energy. Finally, the dry powder treatment allows the powder to be dispensed automatically. This makes it possible to make the transition to automated production, which is especially important when working with fine powders of metals and alloys. Peculiarities of structure formation and its dependence on the parameters of electromagnetic field have been revealed, the sequence of operations has been substantiated. The efficiency of these operations is determined by value of gradient of induction and frequency of alternating magnetic field, induction of permanent magnetic field. The developed mold designs allow efficient use of electromagnetic fields in forming high anisotropy of magnetic properties of powder magnets.

\section{References}

1. P.I. Beloborodov, V.A. Vovodin, V.A. Ignatenko. Basic state of dipole system in flat rectangular center net // Journal of Experimental and Theoretical Physics, Vol. 68, №3, pp. 889-893 (1985)

2. B.E. Levin , Y. D. Tretyakov, L.M. Letyuk. Physicochemical bases of production, properties and use of ferrites, M.: Metallurgia, 427 p. (1979)

3. Y.M. Vernigorov, N.N. Frolova. Destruction of ferromagnetic material particles in magneto-vibrational layer with high strength // Vestnik of Don State Technical University, Vol. 11, №7 (58), pp. 1127-1131 (2011)

4. Y.M. Vernigorov, N.N. Frolova. Collisions of compact ferromagnetic material particles in magneto-vibrational layer // Vestnik of Don State Technical University, №1 (62), pp. 95-98 (2012)

5. A.P. Babichev, Y.M. Vernigorov, N.N. Frolova. Operation modes of fine powder grinding device $\mathrm{SmCo}_{5} / /$ Fundamental and applied problems of technics and technology, № 6 (296). pp. 64-70 (2012)

6. J. Thomson. Instability and disasters in science and technology, M.: Mir, 254 p. (1985)

7. Y.M. Vernigorov,K.K. Leletko. Magneto-vibrational technology of dosing powders of magneto-rigid materials // Vestnik of Don State Technical University, №4 (65), pp. 24$30(2018)$

8. Y.M. Vernigorov, K.K. Leletko, N.N. Frolova. Simulation of destruction of ferromagnetic materials particles in magneto-vibrational layer // World Science Proceedings of articles the international scientific conference, pp. 59-70 (2017)

9. Y.M. Vernigorov. Magneto-vibrational technology of powder magnets production: dissertation for the degree of D Eng., Rostov-on-Don, 369 p. (1995) 